\title{
Degeneration of Myelinated Efferent Fibers Induces Spontaneous Activity in Uninjured C-Fiber Afferents
}

\author{
Gang Wu, ${ }^{1}$ Matthias Ringkamp, ${ }^{1}$ Beth B. Murinson, ${ }^{2}$ Esther M. Pogatzki, ${ }^{1}$ Timothy V. Hartke, ${ }^{1}$ \\ Himali M. Weerahandi, ${ }^{1}$ James N. Campbell, ${ }^{1,4}$ John W. Griffin, ${ }^{2,3}$ and Richard A. Meyer ${ }^{1,4}$ \\ Departments of ${ }^{1}$ Neurosurgery, ${ }^{2}$ Neurology, and ${ }^{3}$ Neuroscience, and the ${ }^{4}$ Applied Physics Laboratory, Johns Hopkins \\ University, Baltimore, Maryland 21287
}

\begin{abstract}
We demonstrated recently that uninjured C-fiber nociceptors in the L4 spinal nerve develop spontaneous activity after transection of the L5 spinal nerve. We postulated that Wallerian degeneration leads to an alteration in the properties of the neighboring, uninjured afferents from adjacent spinal nerves. To explore the role of degeneration of myelinated versus unmyelinated fibers, we investigated the effects of an L5 ventral rhizotomy in rat. This lesion leads to degeneration predominantly in myelinated fibers. Mechanical paw-withdrawal thresholds were assessed with von Frey hairs, and teased-fiber techniques were used to record from single C-fiber afferents in the L4 spinal nerve. Behavioral and electrophysiological data were collected in a blinded manner. Seven days after surgery, a marked decrease in withdrawal thresholds was observed after the ventral rhizotomy but not after the sham operation. Single fiber recordings revealed low-frequency spontaneous activity in $25 \%$ of the C-fiber afferents 8-10 d after the lesion compared with only
\end{abstract}

$11 \%$ after sham operation. Paw-withdrawal thresholds were inversely correlated with the incidence of spontaneous activity in high-threshold C-fiber afferents. In normal animals, lowfrequency electrocutaneous stimulation at C-fiber, but not A-fiber, strength produced behavioral signs of secondary mechanical hyperalgesia on the paw. These results suggest that degeneration in myelinated efferent fibers is sufficient to induce spontaneous activity in C-fiber afferents and behavioral signs of mechanical hyperalgesia. Ectopic spontaneous activity from injured afferents was not required for the development of the neuropathic pain behavior. These results provide additional evidence for a role of Wallerian degeneration in neuropathic pain.

Key words: neuropathic pain; nerve injury; sensitization; hyperalgesia; neuropathy; Wallerian degeneration; unmyelinated cutaneous afferent; in vivo; single nerve fiber recording
Pain is a devastating outcome associated with certain neuropathies and traumatic nerve injuries. A number of animal models have been developed to investigate the mechanisms of neuropathic pain (Bennett and Xie, 1988; Seltzer et al., 1990; Kim and Chung, 1992; Song et al., 1999). Historically, much attention has been paid to the alterations that occur in the injured neuron. Many studies have demonstrated that ectopic spontaneous activity and mechanosensitivity occur at the nerve injury site and the dorsal root ganglia (DRG) of injured neurons (Blumberg and Jänig, 1984; Meyer et al., 1985; Koschorke et al., 1991; Proske et al., 1995; Liu et al., 2000). In addition, the expression of molecules related to nociception changes in injured primary afferents (Noguchi et al., 1995; Fukuoka et al., 1998; Black et al., 1999; Boucher et al., 2000).

However, mounting evidence suggests that important changes also occur in adjacent, uninjured neurons (Gold, 2000). The failure of an L5 dorsal rhizotomy to prevent or reverse the hyperalgesia associated with an L5 spinal nerve ligation indicates that events outside of the injured root play a role (Li et al., 2000). Indeed, unmyelinated, nociceptive afferents from adjacent, uninjured nerve roots develop spontaneous activity within $1 \mathrm{~d}$ of an L5

\footnotetext{
Received Dec. 18, 2001; revised June 10, 2002; accepted June 13, 2002.

This research was supported by the Johns Hopkins Blaustein Pain Research Fund and National Institutes of Health Grants NS 41269 and NS 14447. We greatly appreciate the assistance of Dr. Yongbo Li.

Correspondence should be addressed to Richard Meyer, 5-109 Meyer Building, Department of Neurosurgery, Johns Hopkins University School of Medicine, 600 North Wolfe Street, Baltimore, MD 21287. E-mail: rmeyer@jhmi.edu.

Copyright (C) 2002 Society for Neuroscience $0270-6474 / 02 / 227746-08 \$ 15.00 / 0$
}

spinal nerve injury (Wu et al., 2001). In addition, the cutaneous terminals of uninjured nociceptors become responsive to adrenergic agents (Sato and Perl, 1991; Ali et al., 1999) and sensitized to mechanical and heat stimuli (Shim et al., 2001). Also, various molecules associated with nociception are upregulated in the L4 DRG after an L5 spinal nerve injury (Fukuoka et al., 1998, 2000a,b; Porreca et al., 1999; Boucher et al., 2000; Xie et al., 2000; Hudson et al., 2001; Kim et al., 2001).

One possible explanation for these observations is that Wallerian degeneration of the injured fibers in the peripheral nerve leads to changes in adjacent, uninjured fibers. Indeed, several authors have reported previously a role for Wallerian degeneration in neuropathic pain (Myers et al., 1996; Ramer et al., 1997; Sommer and Schäfers, 1998). A spinal nerve lesion produces peripheral degeneration in motor as well as sensory nerve fibers and therefore does not allow an evaluation of the relative role of degeneration in these different classes of nerve fibers. Recently, several groups have shown that a selective lesion to motor fibers in the L5 spinal nerve produces behavioral signs of hyperalgesia (Li et al., 2002; Sheth et al., 2002). In the present study, we therefore sought to determine whether degeneration in motor fibers also leads to the development of spontaneous activity in unmyelinated, uninjured afferents.

\section{MATERIALS AND METHODS}

Experimental animals. Twenty-four male Sprague Dawley rats weighing 200-300 gm were studied. Two to four animals were placed in plastic cages with sawdust bedding and housed in a climate controlled room 
under a 14/10 hr light/dark cycle. The Johns Hopkins University Animal Care and Use Committee approved the experimental protocol.

Surgical procedures for producing the neuropathic pain model. Fourteen animals were randomly assigned to two different surgical groups, ventral rhizotomy or sham. Anesthesia was initiated with pentobarbital (50 $\mathrm{mg} / \mathrm{kg}$, i.p.) and maintained with supplemental doses as needed. Animals were given a prophylactic dose of antibiotics to prevent infections (Bicillin, 100,000 U/kg, i.m.; Wyeth Laboratories, Philadelphia, PA). After a midline skin incision in the lumbar region, the L5 lamina was freed of its muscular attachments, and an L5 hemilaminectomy was performed under an operating microscope. The base of the transverse process of the L5 vertebra was partially removed to obtain a good exposure of the L5 ventral root. The dura and arachnoid were incised, and the L5 ventral root was identified as it lies just beneath the dorsal root. The ventral root was transected 3-4 $\mathrm{mm}$ rostral to the $\mathrm{L} 5 \mathrm{DRG}$, and $\sim 1 \mathrm{~mm}$ of the root was removed. Great effort was taken to avoid any damage to the L5 dorsal roots and its DRG. The L5 DRG was not exposed during the hemilaminectomy. For the sham-operated animals, the dura and arachnoid were incised, and the ventral root was exposed but not cut.

Two animals were prepared in a given surgical session, one sham and one lesioned. In subsequent behavioral and electrophysiological experiments, the experimenters were blinded as to which animal was being tested to prevent bias. The experimenter performing the behavioral assay was not informed about the ongoing protocol and therefore did not know the type of lesion. Furthermore, this experimenter was not involved in the electrophysiological experiments. In the electrophysiological experiments, the person who anesthetized and prepared the animals for the electrophysiological recordings was not involved in collecting data. Because both the sham-operated and lesioned animals had similar operative procedures, the scar tissue that was observable at the time of recording was not different and could not be used as a cue by the experimenters performing the electrophysiological recordings. In addition, no obvious wasting of muscles was apparent. At the end of the experiments on each pair of animals, an autopsy was conducted to confirm that the appropriate lesion was performed.

Behavioral testing with mechanical stimuli. The rats were tested two times preoperatively and at 4 and $7 \mathrm{~d}$ postoperatively. Testing was performed at approximately the same time during the day.

To quantify mechanical sensitivity of the foot, paw-withdrawal threshold was measured using the up-down testing paradigm (Dixon, 1980; Chaplan et al., 1994) with a series of von Frey probes $(3.2,5.2,8.3,15,29$, 44, 64, 94, and $160 \mathrm{mN}$ ). Two rats (one sham-operated and one lesioned animal) were placed under separate transparent Plexiglas chambers positioned on a wire mesh floor. Five minutes were allotted for habituation. Each stimulus consisted of a 2-3 sec application of the von Frey probe to the middle of the plantar surface of the foot with a 1-2 min interval between stimuli. Quick withdrawal or licking of the paw in response to the stimulus was considered a positive response. Threshold values were derived according to the method described by Chaplan et al. (1994). If the animal showed no response to the von Frey probes, a value of $260 \mathrm{mN}$ was assigned to threshold (Ringkamp et al., 1999).

Electrophysiological procedures. Electrophysiological recordings were performed 8-10 d after surgery. The rats were initially anesthetized with pentobarbital $(50 \mathrm{mg} / \mathrm{kg}$, i.p. $)$. The jugular vein was cannulated for subsequent intravenous administration of pentobarbital $\left(8-15 \mathrm{mg} \cdot \mathrm{kg}^{-1}\right.$. $\mathrm{hr}^{-1}$ ) to maintain anesthesia. Heart rate was continuously monitored by an electrocardiogram and used as an indicator of adequate anesthesia. A tracheotomy was performed, and animals were artificially ventilated with a mixture (1:3) of oxygen and room air to maintain expired $\mathrm{pCO}_{2}$ at 40 $\mathrm{mmHg}$. Muscle relaxation was induced by an intravenous dose of pancuronium bromide $(1 \mathrm{mg} / \mathrm{kg})$ and maintained by supplemental doses hourly. Animal core temperature was measured by a rectal probe and maintained at $37-38^{\circ} \mathrm{C}$ using feedback-controlled, water-perfused heating pads.

Electrophysiological recordings were made from the L4 spinal nerve (Fig. 1A). Teased-fiber recording techniques were used as described previously (Campbell and Meyer, 1983). Briefly, the L4 spinal nerve was carefully exposed. Small bundles were cut from the L4 spinal nerve and teased into small filaments suitable for recording activity from single nerve fibers. The neural signal was differentially amplified, filtered, and digitized at a rate of $25 \mathrm{kHz}$. A real-time computer-based data acquisition and processing system (DAPSYS; Brian Turnquist, Johns Hopkins University) provided multiple window discriminators for real-time sorting of different action potential (AP) waveforms (for details, go to http://www.dapsys.net). In addition, all waveforms passing a selectable threshold level were saved for post hoc analysis.
A

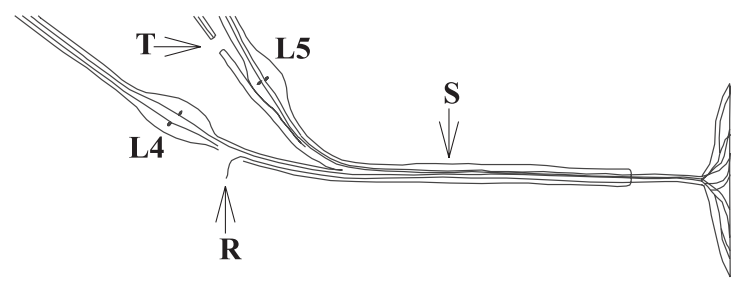

B

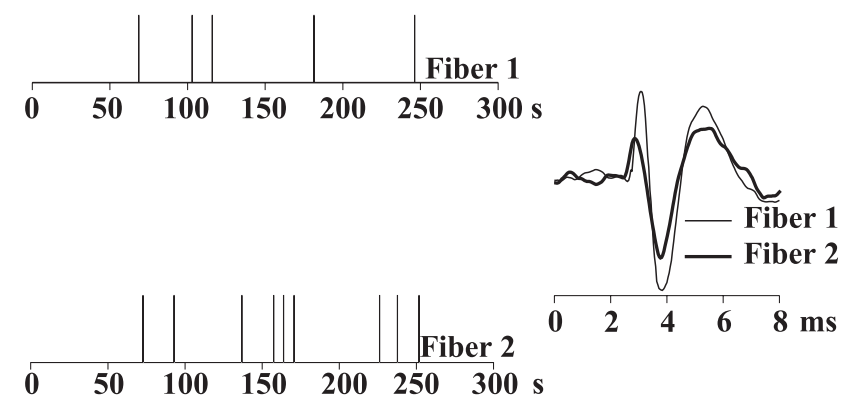

C

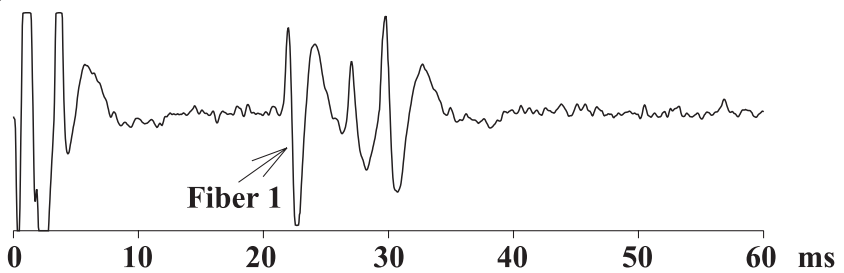

Figure 1. Spontaneous activity in two typical C-fiber afferents recorded $8 \mathrm{~d}$ after an L5 ventral rhizotomy. $A$, Teased-fiber techniques were used to record activity from single nerve fibers of the L4 spinal nerve $(R)$ in sham-operated animals and in animals after transection of the L5 ventral root $(T)$. Electrical stimulation of the sciatic nerve $(S)$ was used to identify and count the number of $\mathrm{C}$-fibers at the recording electrode. $B$, The presence of spontaneous action potential activity was assessed over a 5 min recording interval. In this example, two $\mathrm{C}$-fibers with low-grade spontaneous activity were recorded simultaneously. On the left, each panel represents the spontaneous activity of one fiber, and each vertical line corresponds to the time of occurrence of an action potential. The action potential waveforms are illustrated on the right. $C$, Suprathreshold electrical stimulation at the sciatic nerve produced three discrete action potential waveforms at $\mathrm{C}$-fiber latencies. The action potential waveform starting at $21 \mathrm{msec}$ (peak at $22 \mathrm{msec}$ ) had the same shape as the spontaneously active fiber 1 , providing evidence that the spontaneous activity came from this $\mathrm{C}$-fiber. The two other $\mathrm{C}$-fibers had superimposed waveforms (from 26 to $32 \mathrm{msec}$ ). Fiber 2 corresponds to one of these $\mathrm{C}$-fibers.

The sciatic nerve was exposed, and a stimulating electrode was placed under the nerve, $\sim 2 \mathrm{~cm}$ distal to the recording electrode. The number of $\mathrm{C}$-fiber waveforms observed at the recording electrode was determined in response to electrical stimulation of the sciatic nerve (one pulse every $4 \mathrm{sec}$ ). The stimulus strength was gradually increased, and each new $\mathrm{C}$-fiber waveform was counted. These numbers were used to estimate the proportion of spontaneously active C-fibers. Spontaneous activity was recorded for at least $5 \mathrm{~min}$.

Great care was taken to ensure positive identification of spontaneously active C-fibers and to avoid incorrect classification of artifacts. Only filaments with a good signal-to-noise ratio $(>4: 1)$ were used. In addition, the bundles were finely dissected to keep the number of $\mathrm{C}$-fibers in the filaments low (median, three; range, one to six $\mathrm{C}$-fibers per filament). For many of the spontaneously active C-fibers (Fig. 1, fiber 1), the action potential waveform matched one of the waveforms elicited by electrical stimulation. However, a match was not always possible when the electrically evoked waveform of the spontaneously active fiber overlapped with the waveform of another C-fiber (Fig. 1, fiber 2).

Ongoing activity in A-fibers, consisting mainly of muscle afferents, was 
frequently observed from both normal and lesioned animals. The ongoing A-fiber activity was usually at a fairly high frequency. This prohibited an accurate $\mathrm{C}$-fiber conduction velocity survey attributable to interference between spontaneous A-fiber waveforms and electrically evoked $\mathrm{C}$-fiber waveforms. Therefore, the analysis of the incidence of $\mathrm{C}$-fiber spontaneous activity was restricted to those filaments in which A-fiber spontaneous activity was absent or present at a very low frequency.

In an attempt to sample throughout the L4 spinal nerve, only two filaments were recorded from any bundle that was dissected from the nerve. After two filaments were recorded, a new bundle was taken from the nerve trunk. By visual estimation, each bundle represented $<5 \%$ of the nerve, and each filament represented $<5 \%$ of a bundle. By the end of the recording session, at least half of the nerve was studied.

As a result of these stringent criteria for choosing filaments for recording, $<50 \%$ of the filaments were actually studied. However, in our typical recording sessions, we were able to record from an average of 11 filaments for each animal (range, 6-16 filaments). Because all experiments were blinded, the criteria were the same for lesioned and shamoperated animals.

Histological techniques. Animals were killed by cardiac puncture under deep anesthesia and subsequently perfused with buffered saline and fixative. Fixation was for a total of $24 \mathrm{hr}$ with $4 \%$ paraformaldehyde and $3 \%$ glutaraldehyde in Sorenson's buffered saline. Preparation of tissues for light and electron microscopy (EM) was performed, including washing with buffer, osmium fixation $\left(1 \% \mathrm{OsO}_{4}\right.$ for $45 \mathrm{~min}$ at room temperature), dehydration, and standard plastic embedding. For light microscopy, $1-\mu \mathrm{m}$-thick sections were stained with Toluidine blue. Light microscope images were photomicrographed using a Nikon (Tokyo, Japan) Fujix HC 300Zi digital camera. For EM, silver thin sections of $\sim 80 \mathrm{~nm}$ thickness were stained with uranyl acetate and lead citrate. Tissues suspended on Formvar-coated (Electron Microscopy Sciences, Fort Washington, PA) gold grids were viewed and photomicrographed using a Hitachi (Tokyo, Japan) 600A electron microscope.

Electrocutaneous stimulation experiments. As will be presented in Results, low-frequency spontaneous activity was evident in L4 C-fibers recorded from lesioned rats. To assess whether low-frequency activity in C-fiber afferents could lead to behavioral signs of hyperalgesia, we electrically stimulated the heel of eight naive rats and tested for mechanical responsiveness to von Frey probes. A stimulating electrode $(5 \mathrm{~mm}$ diameter with electroconducting gel) was placed on the heel, and the return electrode $(15 \times 8 \mathrm{~mm})$ was placed on the dorsum of the same foot. Under isoflurane anesthesia $(2.0-2.5 \%)$, the foot was electrically stimulated (1 msec duration) every $5 \mathrm{sec}$ for $10 \mathrm{~min}$ (yielding 120 pulses) at either $50 \%$ above the threshold current to achieve muscle twitching (A-fiber stimulation, $0.63 \pm 0.08 \mathrm{~mA}$ ) or at $100 \mathrm{~mA}$ (C-fiber stimulation). The anesthesia was stopped immediately at the end of the electrical stimulation, and the animals recovered within $5 \mathrm{~min}$. The stimulated hindpaw (left vs right) and stimulus intensity were randomly selected. The incidence of paw withdrawal to five applications of two different von Frey filaments $(75$ and $150 \mathrm{mN})$ to an area between the footpads was determined on both hind feet three times before electrical stimulation and at various times after stimulation (for times, see Fig. 4). Each animal was stimulated and tested again $4 \mathrm{~d}$ later. For the second test, the opposite foot was stimulated, and the other intensity was used. The experimenter collecting the behavioral data was blinded to stimulation side and intensity.

Statistical analysis. We used parametric and nonparametric analyses where appropriate. Because the von Frey threshold measurements are not normally distributed attributable to the ceiling effect associated with limiting the maximum force that can be used, data are analyzed nonparametrically and presented as median [25\%, 75\% quartiles]. Wilcoxon matched pairs, Mann-Whitney $U, \chi^{2}$, ANOVA, and paired $t$ tests were used.

\section{RESULTS}

Combined behavioral and electrophysiological experiments were performed on 14 animals, on seven animals after transection of the L5 ventral root, and on seven sham-operated controls. The presence of spontaneous action potential activity in C-fibers was assessed over a 5 min recording interval. Fibers in which spontaneous activity was stopped by gentle warming of the skin with a heat lamp were considered to be cold fibers (Leem et al., 1993) and were excluded from this analysis.
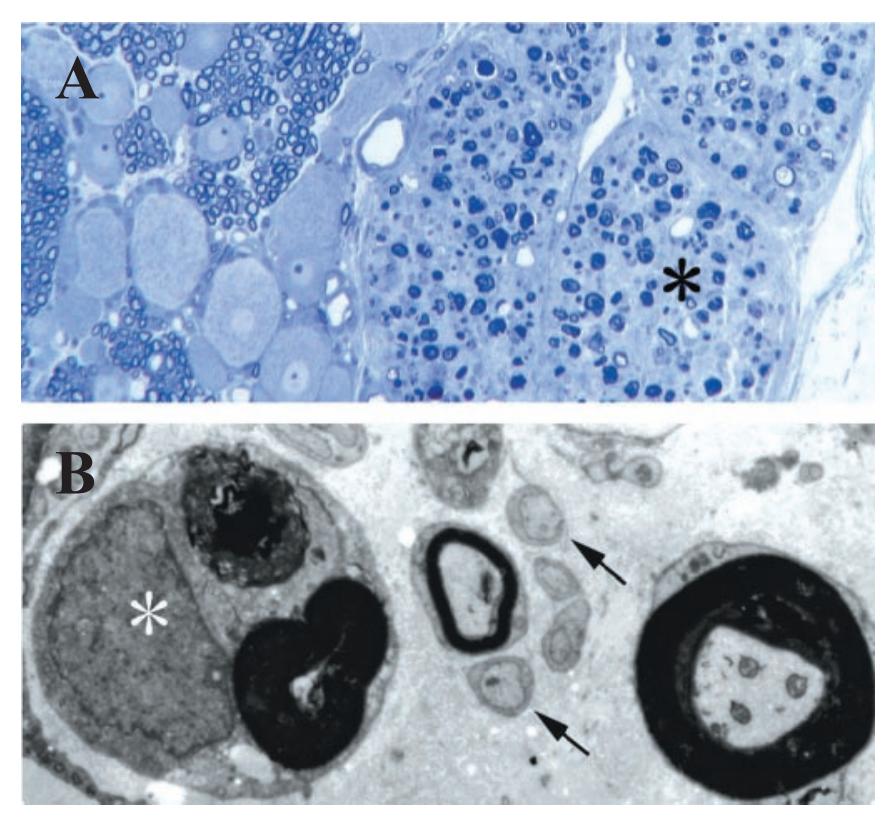

Figure 2. Histological confirmation of the ventral rhizotomy lesion. $A$, Spinal roots at the level of the dorsal root ganglion $7 \mathrm{~d}$ after ventral rhizotomy, demonstrating a selective lesion of the ventral root axons. The ventral root on the right (asterisk) shows ongoing Wallerian degeneration. The dorsal root ganglion on the left shows intermixed cells and axons of the DRG, with no evidence of injury or fiber degeneration. Magnification, $100 \times . B$, Left lateral plantar nerve $7 \mathrm{~d}$ after ventral rhizotomy shows a mixture of degenerating and intact myelinated axons. A myelinated axon undergoing Wallerian degeneration (asterisk) is seen in close proximity to the unmyelinated axons of C-fibers (arrows), a small myelinated axon, and an intact large myelinated axon (on the right). Magnification, 4500×.

\section{Ventral rhizotomy induces Wallerian degeneration in some myelinated axons}

The ventral rhizotomy led to degeneration that was restricted to axons arising from the ventral root (Fig. $2 A$ ). At $7 \mathrm{~d}$ after the ventral rhizotomy, a mixture of degenerating and intact axons was observed along the entire length of the nerve distal to the axotomy, extending from the L5 spinal nerve to the plantar nerves of the hindpaw. Wallerian degeneration of myelinated axons occurred in close proximity to C-fibers (Fig. 2B).

\section{Ventral rhizotomy produces behavioral signs of hyperalgesia}

Paw-withdrawal thresholds decreased significantly after the ventral rhizotomy. Thresholds on the side ipsilateral to the L5 ventral rhizotomy dropped from $260[260,260] \mathrm{mN}$ before the lesion to $29[21,49] \mathrm{mN}$ after the lesion $(p \leq 0.05$; Wilcoxon matched pairs) (Fig. 3A). These thresholds after the lesion were also lower than the thresholds on the ipsilateral paw of the sham-operated animals $(260[260,260] \mathrm{mN} ; p \leq 0.01$; Mann-Whitney $U$ test $)$. No significant changes in paw-withdrawal thresholds were observed in the contralateral foot of the lesioned or sham-treated animals.

\section{Spontaneous activity develops in uninjured C-fibers of spinal nerve L4}

Low-grade spontaneous activity developed after the L5 ventral rhizotomy. Examples of two spontaneously active C-fibers, recorded simultaneously from one filament, are shown in Figure $1 B$. These recordings were obtained $8 \mathrm{~d}$ after an L5 ventral rhizotomy. Low-grade spontaneous activity was observed in both fibers (5 APs/5 min for fiber 1 and 9 APs/5 min for fiber 2). Electrical stimulation of the sciatic nerve demonstrated the pres- 

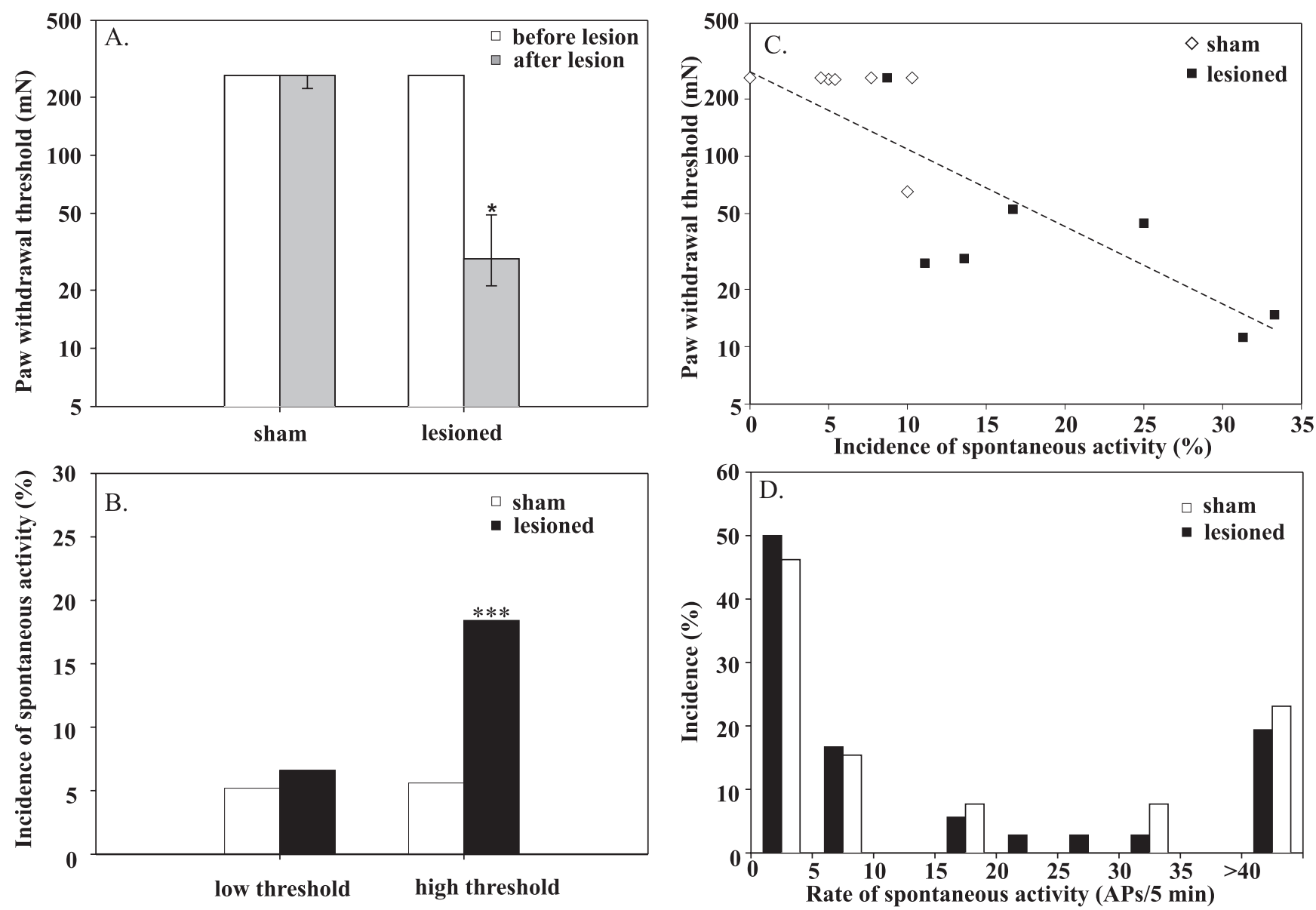

Figure 3. Ventral rhizotomy produces mechanical hyperalgesia on the side ipsilateral to the lesion and spontaneous activity in C-fibers. $A$, Mechanical withdrawal thresholds decreased after the lesion but not after the sham surgery. Measurements were made before (open bars) and $7 \mathrm{~d}$ after ( filled bars) the surgery $\left({ }^{*} p<0.05\right.$; Wilcoxon matched pairs test). $B$, Incidence of spontaneous activity in high-threshold C-fibers increases after the L5 ventral rhizotomy $\left({ }^{* * *} p<0.001 ; \chi^{2}\right.$ test). The proportion of low-threshold fibers with spontaneous activity that responded to gentle brushing of the skin did not change with the lesion. However, the proportion of high-threshold fibers with spontaneous activity that did not respond to gentle brushing of the skin significantly increased after the lesion. $C$, Hyperalgesia is associated with a high incidence of spontaneous activity. The paw-withdrawal threshold for each animal is plotted as a function of the incidence of spontaneous activity in high-threshold C-fiber afferents for that animal. Animals with low paw-withdrawal thresholds had a higher incidence of spontaneous activity in high-threshold C-fiber afferents. Open symbols correspond to sham-operated animals, and filled symbols correspond to lesioned animals. $D$, Distribution of discharge frequencies for the high-threshold, C-fiber afferents. The distribution for the lesioned animals ( filled bars) was similar to the distribution for the sham-operated animals (open bars). Data are normalized by the total number of spontaneously active, high-threshold afferents $(n=36$, lesioned; $n=13$, sham). The experimenters were blinded to the type of surgery at the time of the behavioral and electrophysiological measurements. Seven sham-operated animals and seven lesioned animals were studied.

ence of three $\mathrm{C}$-fibers on the recording electrode (Fig. 1C). One of these $\mathrm{C}$-fibers had an action potential waveform that matched the action potential waveform of fiber 1 (conduction latency, 21 msec; conduction velocity, $1.05 \mathrm{~m} / \mathrm{sec}$ ). One of the other two $\mathrm{C}$-fibers corresponded to fiber 2; these two C-fibers had similar conduction latencies, and their waveforms are superimposed in Figure $1 C$ (conduction latency, $30 \mathrm{msec}$; conduction velocity, 0.77 $\mathrm{m} / \mathrm{sec}$ ). They could be identified as two units because their electrical thresholds were different.

In the lesioned animals, $25 \%$ of the C-fibers (49 of 194) were spontaneously active. This incidence of spontaneous activity after the L5 ventral rhizotomy was lower than the incidence of spontaneous activity after an L5 spinal nerve ligation-cut reported previously by us (Wu et al., 2001) (46\%; $p<0.001 ; \chi^{2}$ test on proportions). In sham-operated animals, $<11 \%$ of the C-fibers ( 25 of $231 ; p<0.001 ; \chi^{2}$ test compared with lesioned animals) in the L4 spinal nerve were spontaneously active, which is comparable with the number reported previously (Wu et al., 2001) for normal, control animals $\left(8.5 \% ; p>0.1 ; \chi^{2}\right.$ test on proportions).

\section{High-threshold afferents exhibit spontaneous activity}

We did not use noxious stimuli to search for nociceptive receptive fields to avoid sensitization of afferent terminals. However, a light brush was stroked across the skin to identify low-threshold, mechanoreceptive afferents. The proportion of low-threshold C-fibers in the lesioned animals (11\%; 22 of 194 fibers) was not significantly different from the proportion in the sham animals $(15 \% ; 35$ of 231 fibers; $p>0.2 ; \chi^{2}$ test). In addition, the proportion of spontaneously active low-threshold $\mathrm{C}$-fiber afferents was similar in sham-operated animals $(5.2 \%)$ and lesioned animals $(6.6 \%)$ (Fig. 3B).

The fibers without a response to light brushing are presumably high-threshold nociceptive afferents. When the low-threshold fibers are excluded from the analysis, the incidence of spontaneous activity in the remaining high-threshold afferents is more than threefold greater in the lesion animals $(18.4 \%)$ compared with the sham animals $\left(5.6 \% ; p<0.001 ; \chi^{2}\right.$ test on proportions) (Fig. $\left.3 B\right)$.

The distribution of discharge frequencies observed in the spon- 
taneously active, high-threshold C-fiber afferents is shown in Figure $3 D$. The median discharge frequency in these C-fibers was low in the lesioned animals $(5.5[2.5,23.5] \mathrm{APs} / 5 \mathrm{~min})$ and was not significantly different from the sham-operated animals (6.0 [2.8, 35.5] APs/5 min; $p>0.1$; Mann-Whitney $U$ test). The median discharge frequency in the high-threshold $\mathrm{C}$-fiber afferents also did not differ substantially from that seen in the low-threshold afferents $(3.0[2.0,8.0]$ APs/5 min; $p>0.1)$. Many of the highthreshold fibers exhibited an irregular or bursting discharge with a peak instantaneous frequency of 0.69 [0.06, 5.77] APs/sec.

A C-fiber was considered to be spontaneously active if it exhibited one action potential during the 5 min recording period. When a more conservative criterion of five action potentials per 5 min recording period is used, the proportion of high-threshold fibers with spontaneous activity after a lesion (10.2\%) was still significantly greater than for sham-operated animals $(3.0 \% ; p<$ $0.01 ; \chi^{2}$ test on proportions).

\section{Incidence of spontaneous activity is increased in animals with hyperalgesic behavior}

The paw-withdrawal thresholds were inversely correlated with the incidence of spontaneous activity in high-threshold C-fiber afferents (Fig. $3 C)\left(R^{2}=0.72 ; p \leq 0.01\right)$. In other words, the animals that exhibited a low mechanical withdrawal threshold had a higher incidence of spontaneous activity.

\section{Spontaneous activity originating from receptive field of cutaneous nociceptors}

In two additional experiments not included in the incidence analysis, we purposefully searched for the receptive field of spontaneously active $\mathrm{C}$-fibers. The first $\mathrm{C}$-fiber of the day was investigated in each case to avoid the possibility that the intense search stimulus needed to locate and characterize the receptive field of nociceptors contributed to the initial spontaneous activity. Both fibers had cutaneous receptive fields with a high threshold for mechanical stimuli, and thus both would be considered to be nociceptors. In each fiber, the spontaneous activity was eliminated for more than $1 \mathrm{hr}$ by intradermal administration of lidocaine $(1 \%$ in $50 \mu \mathrm{l})$ into the receptive field.

\section{Low-frequency electrocutaneous stimulation produces behavioral signs of hyperalgesia}

In normal animals, electrical stimulation of the heel at C-fiber strength resulted in a significant increase in the incidence of paw withdrawal to the 75 and $150 \mathrm{mN}$ von Frey probes applied ipsilateral to the area between the footpads (Fig. 4). The incidence of paw withdrawal remained elevated for $15 \mathrm{~min}$. A significant increase in the incidence of paw withdrawal was not observed after stimulation at A-fiber strength. In addition, no significant changes were observed in the contralateral foot for either stimulation intensity. Thus, low-frequency (one pulse every $5 \mathrm{sec}$ for $10 \mathrm{~min}$ ) stimulation at $\mathrm{C}$-fiber, but not A-fiber, strength is able to produce behavioral signs of secondary mechanical hyperalgesia.

\section{DISCUSSION}

This is the first study showing that spontaneous activity in uninjured, C-fiber nociceptive afferents develops after a selective injury to efferent myelinated fibers induced by ventral rhizotomy. This injury also induces hyperalgesic behavior. Thus, degeneration in myelinated efferent fibers is sufficient to induce spontaneous activity in C-fiber afferents and behavioral signs of mechanical hyperalgesia. Furthermore, we show that low-frequency

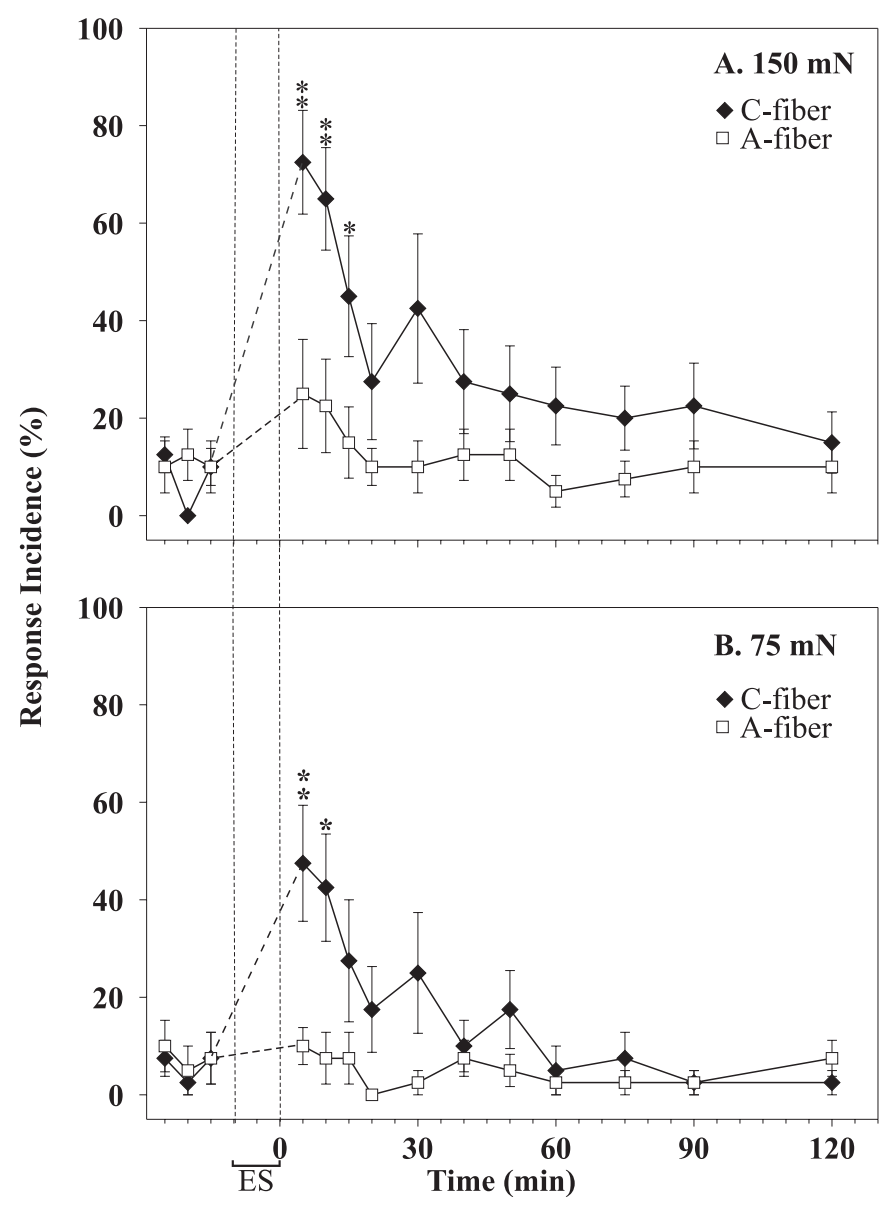

Figure 4. Low-frequency electrocutaneous stimulation at C-fiber strength produces behavioral signs of hyperalgesia. The incidence of paw withdrawal is plotted as a function of time. Electrical stimulation $(E S)$ of the heel ( 1 pulse every $5 \mathrm{sec}$ for $10 \mathrm{~min}$ ) was applied at $\mathrm{C}$-fiber strength (filled symbols) or at A-fiber strength (open symbols). A, The response to the $150 \mathrm{mN}$ von Frey probe increased significantly immediately after the termination of the $\mathrm{C}$-fiber stimulation and remained high for $15 \mathrm{~min}$. $B$, The response to the $75 \mathrm{mN}$ probe also increased significantly after the $\mathrm{C}$-fiber stimulation. There was no significant change in response after the A-fiber stimulation. ( $* p \leq 0.05$ and $* * p \leq 0.01$ with respect to average baseline response; ANOVA, followed by paired $t$ test)

electrical stimulation of $\mathrm{C}$-fibers is able to induce mechanical hyperalgesia in naive rats. These results provide additional evidence for a role of Wallerian degeneration in neuropathic pain.

\section{Ectopic activity in uninjured axons}

The spontaneously active L4 afferents in this study were not directly injured because the lesion was to L5 ventral root fibers. We demonstrated previously that spontaneous activity also develops in uninjured $\mathrm{C}$-fiber afferents after a spinal nerve lesion in the rat (Wu et al., 2001) and monkey (Ali et al., 1999). The incidence of spontaneous activity after the L5 ventral rhizotomy (25\%) performed in the current study was lower than that observed previously after spinal nerve injury (46\%) (Wu et al., 2001). This could reflect that fewer nerve fibers are destroyed by the ventral rhizotomy. Alternatively, muscle nociceptors may be more affected by the injury. Muscle nociceptors are likely underrepresented because they commingle with muscle afferents and filaments containing high rates of ongoing activity from muscle spindles could not be studied.

The magnitude of mechanical hyperalgesia after the ventral 
rhizotomy was not substantially different from that observed after a spinal nerve injury (Li et al., 2002; Sheth et al., 2002) despite the lower incidence of spontaneous activity in L4 afferents. However, an L5 ventral rhizotomy may also result in spontaneous activity in L5 C-fiber afferents. The incidence of spontaneous activity in L5 afferents would likely be higher than that observed in L4 afferents because of the closer proximity to axons of the injured L5 ventral root. Alternatively, full-blown hyperalgesic behavior may develop once the ongoing spontaneous activity exceeds a certain level, and thus higher levels of spontaneous activity may not yield lower withdrawal thresholds.

\section{Ectopic activity in injured axons}

Many authors have demonstrated that an injury to a peripheral nerve results in ectopic mechanical, thermal, and chemical sensitivity, as well as spontaneous activity in the injured afferents (Blumberg and Jänig, 1984; Koschorke et al., 1991). Spontaneous activity may develop in myelinated afferents originally innervating muscle and joints (Proske et al., 1995; Michaelis et al., 2000) or in unmyelinated afferents from cutaneous nerves (Blumberg and Jänig, 1984; Meyer et al., 1985). Surprisingly, an injury to the L5 spinal nerve appears to lead to spontaneous active only in A-fiber afferents (Boucher et al., 2000; Liu et al., 2000). However, low-grade spontaneous activity in C-fibers comparable with that reported here may have been missed because of high-frequency discharge in A-fibers or considered insignificant. Spontaneous activity in injured A-fibers is thought to be involved in the development of neuropathic pain. However, transection of the L5 dorsal root before or after an L5 spinal nerve lesion did not abolish neuropathic pain (Eschenfelder et al., 2000; Li et al., 2000). Thus, spontaneous activity from the injured spinal nerve is not essential for producing neuropathic pain.

A ventral rhizotomy leads to peripheral degeneration in myelinated efferent fibers. Although both myelinated and unmyelinated ventral root afferents are thought to exist, their number in the L5 ventral root appears to be quite small $(<4 \%)$ (Coggeshall et al., 1977; Zenker et al., 1979; Nam et al., 1989). Thus, a ventral rhizotomy at the L5 level can be considered a relatively selective injury to myelinated efferent fibers. Therefore, our results provide additional evidence that ectopic activity in injured afferents is not a requirement for the development of neuropathic pain. One could argue that some afferents are injured as part of the surgical preparation (e.g., dorsal cutaneous ramus). However, the sham animals were subjected to the same surgical approach as the lesioned animals, and thus the difference between sham and lesion cannot be explained in this way.

\section{Does low-frequency activity in C-fibers produce hyperalgesia?}

Previous investigations in acute models of central sensitization suggest that selective activation of $\mathrm{C}$-fiber afferents leads to central sensitization (Woolf, 1992; Kilo et al., 1994; Koltzenburg et al., 1994). In these experiments, high discharge rates are presumed. In contrast, the C-fiber afferents in this study exhibited low rates of spontaneous activity (median, $\sim 1.0 \mathrm{AP} / \mathrm{min}$ ). However, a relatively large proportion of the uninjured, C-fiber afferents develops spontaneous activity after a ventral rhizotomy and after a spinal nerve lesion. Thus, low discharge rates may produce central sensitization over time if a sufficient proportion of the C-fiber population is involved.

Low-frequency activity in C-fibers can produce an increase in peripheral blood flow attributable to the release of vasoactive neurotransmitters from peripheral nociceptor terminals (Lynn and Cotsell, 1992; Magerl and Treede, 1996). Thus, high-frequency activity in $\mathrm{C}$-fibers is not required for a pharmacologically relevant release of neuropeptides in the periphery. A similar release of neurotransmitters-modulators from central terminals of C-fiber nociceptors in the dorsal horn could be sufficient for development of central sensitization. This may be even more so under pathological conditions also affecting glial cells. Glial cells are known to modulate neuronal excitability (Carmignoto, 2000), and this function could be affected by peripheral injury. Spinal glial cells are known to show reactions to injury of peripheral nerves or dorsalventral roots (Colburn et al., 1999; Winkelstein et al., 2001).

We provide evidence that low-frequency $(0.2 \mathrm{~Hz})$ electrocutaneous stimulation of the heel at $\mathrm{C}$-fiber, but not A-fiber, strength is able to produce behavioral signs of secondary hyperalgesia in the hindpaw of naive rats. In human subjects, transcutaneous electrical stimulation at high current densities is able to produce areas of secondary hyperalgesia in the volar forearm when the stimulus frequency is $5 \mathrm{~Hz}$ (Koppert et al., 2001). This group has shown recently (M. Schmelz, personal communication) that electrical stimulation with interpulse intervals as long as $20 \mathrm{sec}$ produces a zone of secondary hyperalgesia within $10 \mathrm{~min}$. These findings support the hypothesis that low-frequency activity in $\mathrm{C}$-fibers is able to produce hyperalgesia to mechanical stimuli.

\section{Do injuries to motor fibers lead to neuropathic pain in humans?}

Although the rats developed signs of neuropathic pain after the ventral rhizotomy, it is not clear whether humans also suffer from neuropathic pain after injuries to motor fibers. Pain is not a principal complaint of patients with amyotrophic lateral sclerosis, but myalgesia is reported with polio. Whether these patients have hyperalgesia has not been carefully studied. In addition, these diseases cause a gradual degeneration in ventral root fibers, whereas ventral rhizotomy induces a simultaneous degeneration in all fibers. Recently, we studied five patients after a surgical lesion of the C7 spinal nerve. One of these patients developed neuropathic pain that lasted for several months (Ali et al., 2002). Thus, a "Chung-type" lesion in humans can produce neuropathic pain.

\section{Wallerian degeneration hypothesis}

We postulate that Wallerian degeneration in the peripheral nerve may play an important role in at least some types of neuropathic pain. To explain the neuropathic pain and spontaneous activity in uninjured C-fiber afferents after a spinal nerve lesion, we proposed previously that transection of the L5 spinal nerve produces a population of partially denervated Remak bundles consisting of degenerating unmyelinated axons from the L5 root and intact unmyelinated axons from the L4 root (Wu et al., 2001). Schwann cells of such Remak bundles would synthesize and release growth factors, cytokines, and short-acting intermediates that could affect uninjured afferents sharing the same Remak bundle. Although ventral rhizotomy induces neuropathic pain behavior, it does not lead to partially denervated Remak bundles (B. B. Murinson, personal communication). Thus, neither partial denervation of Remak bundles nor degeneration of cutaneous nerve fibers is a prerequisite for the development of spontaneous activity in uninjured afferents and of neuropathic pain.

In the sciatic nerve, the efferent nerve fibers from the L5 ventral root are in close proximity to afferent fibers from the L4 and L5 roots. Degenerating myelinated fibers in the plantar nerve 
were found to be in close proximity to unmyelinated fibers (Fig. 2). Products associated with degenerating myelinated fibers, reacting Schwann cells, or infiltrating macrophages may diffuse to nearby sensory fibers and alter their properties. For example, within $24 \mathrm{hr}$ of a nerve section, the expression of cytokines such as tumor necrosis factor- $\alpha$ (TNF $\alpha)$, interleukin- $1 \alpha$ (IL-1 $\alpha)$, IL$1 \beta$, and IL-6 increases in the distal peripheral nerve (Shamash et al., 2002; Stoll et al., 2002). Exogenous administration of TNF $\alpha$ to the peripheral nerve produced activity in $\mathrm{C}$-fiber nociceptors (Sorkin et al., 1997); therefore, TNF $\alpha$ could be a culprit. A ventral rhizotomy has been reported to enhance regeneration of uninjured sensory neurons (Rich and Johnson, 1985).

These results demonstrate that degeneration in myelinated fibers is sufficient to induce spontaneous activity in C-fiber afferents and behavioral signs of mechanical hyperalgesia. In these experiments, the injured fibers were not part of the sensory system. Thus, ectopic spontaneous activity from injured afferents is not required for the development of neuropathic pain behavior. An understanding of the mechanisms that lead to the development of spontaneous activity in uninjured $\mathrm{C}$ fibers may result in novel strategies for the treatment of neuropathic pain.

\section{REFERENCES}

Ali Z, Ringkamp M, Hartke TV, Chien HF, Flavahan NA, Campbell JN, Meyer RA (1999) Uninjured C-fiber nociceptors develop spontaneous activity and alpha adrenergic sensitivity following L6 spinal nerve ligation in the monkey. J Neurophysiol 81:455-466.

Ali Z, Meyer RA, Belzberg AJ (2002) Neuropathic pain after C7 spinal nerve transection in man. Pain 96:41-47.

Bennett GJ, Xie Y-K (1988) A peripheral mononeuropathy in rat that produces disorders of pain sensation like those seen in man. Pain 33:87-107.

Black JA, Cummins TR, Plumpton C, Chen YH, Hormuzdiar W, Clare JJ, Waxman SG (1999) Upregulation of a silent sodium channel after peripheral, but not central, nerve injury in DRG neurons. J Neurophysiol 82:2776-2785.

Blumberg H, Jänig W (1984) Discharge pattern of afferent fibers from a neuroma. Pain 20:335-353.

Boucher TJ, Okuse K, Bennett DL, Munson JB, Wood JN, McMahon SB (2000) Potent analgesic effects of GDNF in neuropathic pain states. Science 290:124-127.

Campbell JN, Meyer RA (1983) Sensitization of unmyelinated nociceptive afferents in the monkey varies with skin type. J Neurophysiol 49:98-110.

Carmignoto G (2000) Reciprocal communication systems between astrocytes and neurones. Prog Neurobiol 62:561-581.

Chaplan SR, Bach FW, Pogrel JW, Chung JM, Yaksh TL (1994) Quantitative assessment of tactile allodynia in the rat paw. J Neurosci Methods 53:55-63.

Coggeshall RE, Emery DG, Ito H, Maynard CW (1977) Unmyelinated and small myelinated axons in rat ventral roots. J Comp Neurol 173:175-184.

Colburn RW, Rickman AJ, DeLeo JA (1999) The effect of site and type of nerve injury on spinal glial activation and neuropathic pain behavior. Exp Neurol 157:289-304.

Dixon WJ (1980) Efficient analysis of experimental observations. Annu Rev Pharmacol Toxicol 20:441-462.

Eschenfelder S, Häbler H-J, Jänig W (2000) Dorsal root section elicits signs of neuropathic pain rather than reversing them in rats with L5 spinal nerve injury. Pain 87:213-219.

Fukuoka T, Tokunaga A, Kondo E, Miki K, Tachibana T, Noguchi K (1998) Change in mRNAs for neuropeptides and the GABA(A) receptor in dorsal root ganglion neurons in a rat experimental neuropathic pain model. Pain 78:13-26.

Fukuoka T, Kondo E, Dai Y, Noguchi K (2000a) Phenotypical change of the L4 DRG neurons after unilateral L5 spinal nerve ligation and the contribution of neurotrophic factors: BDNF increases in TrkA neurons. Soc Neurosci Abstr 26:943.

Fukuoka T, Tokunaga A, Kondo E, Noguchi K (2000b) The role of neighboring intact dorsal root ganglion neurons in a rat neuropathic pain model. In: Progress in pain research and management (Devor M, Rowbotham M, Wiesenfeld-Hallin Z, eds), pp 137-146. Seattle: ISAP.

Gold MS (2000) Spinal nerve ligation: what to blame for the pain and why. Pain 84:117-120.

Hudson LJ, Bevan S, Wotherspoon G, Gentry C, Fox A, Winter J (2001)
VR1 protein expression increases in undamaged DRG neurons after partial nerve injury. Eur J Neurosci 13:2105-2114.

Kilo S, Schmelz M, Koltzenburg M, Handwerker HO (1994) Different patterns of hyperalgesia induced by experimental inflammation in human skin. Brain 117:385-396.

Kim CH, Oh Y, Chung JM, Chung K (2001) The changes in expression of three subtypes of TTX sensitive sodium channels in sensory neurons after spinal nerve ligation. Brain Res Mol Brain Res 95:153-161.

Kim SH, Chung JM (1992) An experimental model for peripheral neuropathy produced by segmental spinal nerve ligation in the rat. Pain 50:355-363.

Koltzenburg M, Torebjörk HE, Wahren LK (1994) Nociceptor modulated central sensitization causes mechanical hyperalgesia in acute chemogenic and chronic neuropathic pain. Brain 117:579-591.

Koppert W, Dern SK, Sittl R, Albrecht S, Schuttler J, Schmelz M (2001) A new model of electrically evoked pain and hyperalgesia in human skin: the effects of intravenous alfentanil, $\mathrm{S}(+)$-ketamine, and lidocaine. Anesthesiology 95:395-402.

Koschorke GM, Meyer RA, Tillman DB, Campbell JN (1991) Ectopic excitability of injured nerves in monkey: entrained responses to vibratory stimuli. J Neurophysiol 65:693-701.

Leem JW, Willis WD, Chung JM (1993) Cutaneous sensory receptors in the rat foot. J Neurophysiol 69:1684-1699.

Li L, Xian CJ, Zhong JH, Zhou XF (2002) Effect of lumbar 5 ventral root transection on pain behaviors: a novel rat model for neuropathic pain without axotomy of primary sensory neurons. Exp Neurol 175:23-34.

Li Y, Dorsi MJ, Meyer RA, Belzberg AJ (2000) Mechanical hyperalgesia after an L5 spinal nerve lesion in the rat is not dependent on input from injured nerve fibers. Pain 85:493-502.

Liu CN, Wall PD, Ben Dor E, Michaelis M, Amir R, Devor M (2000) Tactile allodynia in the absence of $\mathrm{C}$-fiber activation: altered firing properties of DRG neurons following spinal nerve injury. Pain 85:503-521.

Lynn B, Cotsell B (1992) Blood flow increases in the skin of the anaesthetized rat that follow antidromic sensory nerve stimulation and strong mechanical stimulation. Neurosci Lett 137:249-252.

Magerl W, Treede RD (1996) Heat-evoked vasodilatation in human hairy skin: Axon reflexes due to low-level activity of nociceptive afferents. J Physiol (Lond) 497:837-848.

Meyer RA, Raja SN, Campbell JN, Mackinnon SE, Dellon AL (1985) Neural activity originating from a neuroma in the baboon. Brain Res 325:255-260.

Michaelis M, Liu X, Jänig W (2000) Axotomized and intact muscle afferents but not skin afferents develop ongoing discharges of dorsal root ganglion origin after peripheral nerve lesion. J Neurosci 20:2742-2748.

Myers RR, Heckman HM, Rodriguez M (1996) Reduced hyperalgesia in nerve-injured WLD mice: relationship to nerve fiber phagocytosis, axonal degeneration, and regeneration in normal mice. Exp Neurol 141:94-101

Nam SC, Kim KJ, Leem JW, Chung KS, Chung JM (1989) Fiber counts at multiple sites along the rat ventral root after neonatal peripheral neurectomy or dorsal rhizotomy. J Comp Neurol 290:336-342.

Noguchi K, Kawai Y, Fukuoka T, Senba E, Miki K (1995) Substance P induced by peripheral nerve injury in primary afferent sensory neurons and its effect on dorsal column nucleus neurons. J Neurosci 15:7633-7643.

Porreca F, Lai J, Bian D, Wegert S, Ossipov MH, Eglen RM, Kassotakis L, Novakovic S, Rabert DK, Sangameswaran L, Hunter JC (1999) A comparison of the potential role of the tetrodotoxin-insensitive sodium channels, PN3/SNS and NaN/SNS2, in rat models of chronic pain. Proc Natl Acad Sci USA 96:7640-7644.

Proske U, Iggo A, Luff AR (1995) Mechanical sensitivity of regenerating myelinated skin and muscle afferents in the cat. Exp Brain Res 104:89-98.

Ramer MS, French GD, Bisby MA (1997) Wallerian degeneration is required for both neuropathic pain and sympathetic sprouting into the DRG. Pain 72:71-78.

Rich KM, Johnson Jr EM (1985) Ventral rhizotomy enhances regeneration of uninjured sensory neurons. Brain Res 335:182-187.

Ringkamp M, Grethel EJ, Choi Y, Meyer RA, Raja SN (1999) Mechanical hyperalgesia after spinal nerve ligation in rat is not reversed by intraplantar or systemic administration of adrenergic antagonists. Pain 79:135-141.

Sato J, Perl ER (1991) Adrenergic excitation of cutaneous pain receptors induced by peripheral nerve injury. Science 251:1608-1610.

Seltzer Z, Dubner R, Shir Y (1990) A novel behavioral model of neuropathic pain disorders produced in rats by partial sciatic nerve injury. Pain 43:205-218.

Shamash S, Reichert F, Rotshenker S (2002) The cytokine network of Wallerian degeneration: tumor necrosis factor-alpha, interleukin1alpha, and interleukin-1beta. J Neurosci 22:3052-3060.

Sheth RN, Dorsi MJ, Li Y, Murinson BB, Belzberg AJ, Griffin JW, Meyer 
RA (2002) Mechanical hyperalgesia after an L5 ventral rhizotomy or an L5 ganglionectomy in the rat. Pain 96:63-72.

Shim B, Leem JW, Nam TS, Paik KS (2001) Mechanical and heat sensitization of cutaneous nociceptors after peripheral nerve injury in the rat. Soc Neurosci Abstr 27:928:14.

Sommer C, Schäfers M (1998) Painful mononeuropathy in C57BL/Wld mice with delayed Wallerian degeneration: differential effects of cytokine production and nerve regeneration on thermal and mechanical hypersensitivity. Brain Res 784:154-162.

Song XJ, Hu SJ, Greenquist KW, Zhang JM, LaMotte RH (1999) Mechanical and thermal hyperalgesia and ectopic neuronal discharge after chronic compression of dorsal root ganglia. J Neurophysiol 82:3347-3358

Sorkin LS, Xiao W-H, Wagner R, Myers RR (1997) Tumour necrosis factor- $\alpha$ induces ectopic activity in nociceptive primary afferent fibres. Neuroscience 81:255-262.

Stoll G, Jander S, Myers RR (2002) Degeneration and regeneration of the peripheral nervous system: from Augustus Waller's observations to neuroinflammation. J Peripher Nerv Syst 7:13-27.
Winkelstein BA, Rutkowski MD, Sweitzer SM, Pahl JL, DeLeo JA (2001) Nerve injury proximal or distal to the DRG induces similar spinal glial activation and selective cytokine expression but differential behavioral responses to pharmacologic treatment. J Comp Neurol 439:127-139.

Woolf CJ (1992) Excitability changes in central neurons following peripheral damage. In: Hyperalgesia and allodynia (Willis Jr WD, ed), pp 221-243. New York: Raven.

Wu G, Ringkamp M, Hartke TV, Murinson BB, Campbell JN, Griffin JW, Meyer RA (2001) Early onset of spontaneous activity in uninjured $\mathrm{C}$-fiber nociceptors after injury to neighboring nerve fibers. J Neurosci 21:RC140(1-5).

Xie J, Chung JM, Chung K (2000) Differential expression of subtypes of alpha-2A adrenoceptor mRNA in sensory neurons after spinal nerve ligation. Soc Neurosci Abstr 26:945.

Zenker W, Stelzig M, Sulzgruber SC, Neumann A (1979) Fiber analysis of human ventral and dorsal roots on the basis of different acetylcholinesterase activity. Acta Anat 103:319-326. 